\title{
Brown Dwarfs as Ejected Stellar Embryos: Observational Perspectives
}

\author{
Bo Reipurth \\ Institute for Astronomy, University of Hawaii, 2680 Woodlawn Drive, \\ Honolulu, HI 96822, USA \\ Cathie Clarke \\ Institute of Astronomy, Madingley Road, Cambridge CB3 OHA, UK
}

\begin{abstract}
We discuss a scenario in which brown dwarfs are formed like stars, except that their full collapse phases are interrupted through dynamical interactions in small multiple systems, leading to the ejection of the lightest member. This disintegration is a stochastic process, often resulting in the expulsion of newborn low mass stars, but when it occurs early enough the ejected stellar embryo will be a substellar object. This process may be so common at early ages that a large fraction of the ubiquitous brown dwarfs could have formed in this manner. Detailed gas dynamical simulations are required in order to better understand the details of the decay of small newborn multiple systems. We discuss the observational consequences of the ejection hypothesis, noting especially the importance of binaries with brown dwarf components as an observational test. Finally, we note that brown dwarfs that have recently been ejected may be so disturbed, by infall from the collapsing core and also by heavy accretion from perturbed circumstellar disks, that traditional spectral and luminosity criteria may fail to identify their substellar nature.
\end{abstract}

\section{Introduction}

Our understanding of the formation of brown dwarfs has long been shrouded in controversy. Two aspects of their formation appear problematical from a theoretical point of view. The first is that brown dwarf masses are at least 10 times smaller than the typical Jeans mass in star forming molecular clouds. Thus any successful theory needs to produce regions of exceedingly high gas density $\left(\sim 10^{7} \mathrm{~cm}^{-3}\right.$ for isothermal gas at $\left.10 \mathrm{~K}\right)$. Secondly, once such proto-brown dwarfs have formed, it is necessary that they avoid substantial further accretion of gas from their environment, in order that they remain as low mass entities.

A variety of scenarios have been proposed that address the density issue. Many of these involve the formation of brown dwarfs within disks. For example, Lin et al. (1998) suggested that the requisite high densities would be produced during the encounter between two massive disks, when a long tidal filament could be flung out, leading to the subsequent formation of a brown dwarf. In loose $\mathrm{T}$ 
associations such encounters are likely to be extremely rare, which is in contrast to the increasing number of brown dwarfs found also in such environments. In the simulations of Bate (2002), however, where stars form in compact groups, such interactions are common and the majority of brown dwarfs form in massive disks that are highly disturbed by dynamical encounters. Li (2002), on the other hand, has shown that brown dwarfs may also form in isolated disks if these are supported by magnetic pressure. By contrast, Padoan \& Nordlund (2002) have argued that brown dwarfs need not form in disks, but that sufficiently high densities may be produced in the shock compressed regions of turbulent flows; this process they term 'turbulent fragmentation' to distinguish it from the above scenarios in which the high densities required to form brown dwarfs are the result of self-gravity.

Once brown dwarfs have been produced by any of the above mechanisms, the remaining problem is to prevent their exceeding the hydrogen burning mass limit due to continued accretion. Recently, Whitworth \& Zinnecker (2002) have suggested that photoionization may erode higher mass gas cores, so that the remnant core is reduced to substellar masses; evidently this mechanism is restricted to regions containing photoionizing OB stars. Otherwise, there are two possibilities - either the statistics of the density field are such that brown dwarfs collapse in isolation, well away from the gas reservoirs that are destined to form stars, or else, if brown dwarfs and stars form from common gas reservoirs, the brown dwarfs must somehow be removed from this environment.

Reipurth \& Clarke (2001) have developed this latter line of argument, appealing to the dynamical interactions that occur within small $\mathrm{N}$ groupings in order to eject brown dwarfs from their natal gas reservoir. The spectacular simulations of Bate (see contribution this volume) illustrate both the propensity of molecular clouds to fragment into small $\mathrm{N}$ groupings, and the way that dynamical interactions indeed eject brown dwarfs.

In this contribution, we review the ejected stellar embryo scenario and its observable consequences, first considering the observational evidence that stars are indeed formed in small $\mathrm{N}$ groupings. We should stress, however, that the implication is not that all brown dwarfs must form this way, but rather that a significant fraction can be expected to be formed in, and ejected from, multiple systems. It is our purpose here to set out the observational discriminants that can be used to assess what fraction of brown dwarfs form in this way. We emphasize that most of these discriminants must be sought in young brown dwarfs: after a few hundred million years, the appearance and kinematics of substellar objects depend only on mass and age, and the particular mechanism that produced them is lost in the mist of time.

\section{Multiplicity of the Youngest Stars}

In a detailed study of 14 driving sources of giant Herbig-Haro flows, Reipurth (2000) found that more than $80 \%$ are binaries, and of these half are higher order systems. Given that such a survey can only under-estimate the true incidence of faint companions, and if all newborn stars go through a phase producing giant outflows, this may then suggest that the typical star forming environment contains several stars. 
Embedded outflow sources are of the order of $10^{5} \mathrm{yr}$ old or less, and it follows that some of these systems must decay to reach the lower observed frequencies at later evolutionary stages. It is well established that non-hierarchical triple systems undergo rapid dynamical evolution and generally decay into a binary and an unbound, escaping third member. The binary system that is formed in this dynamical process is highly eccentric, and given that the triple disintegration is likely to take place while the stars are still actively accreting gas from an infalling envelope, it follows that the circumstellar disks will interact on an orbital timescale, which will lead to shrinkage of the orbit (e.g. Artymowicz $\&$ Lubow 1996). These interactions are again likely to cause cyclic variations in the accretion rate, with consequent pulses in the outflow production, and the giant Herbig-Haro flows may therefore represent a fossil record of the birth and early evolution of binary systems (Reipurth 2000). Altogether, it appears that the generation of giant Herbig-Haro flows, the birth of a binary, and the formation of brown dwarfs may all be different aspects of a single event, namely the dissolution of a small multiple system.

\section{Brown Dwarfs as Ejected Stellar Embryos}

If stars form in small non-hierarchical groups (Larson 1972), then evidence for these initial conditions is rapidly lost due to the dynamical disintegration of such groups. For a non-hierarchical group containing $\mathrm{N}$ stars, then in the limit of small $\mathrm{N}$ (less than a few 10s), the timescale for the group's dissolution is roughly $\mathrm{N}$ crossing times. This means that even young systems such as the Orion Nebula Cluster, where the stellar distribution is very smooth at an age of a few Myr (Bate et al. 1998), could easily have evolved from an ensemble of compact few body groupings (Scally \& Clarke 2002). Traces of small N clustering must instead be sought in younger (Class 0) sources (see Section 2).

The reason that such groupings dissolve is primarily because, as the system evolves, there are occasions when three stars pass close to another (i.e. close enough that their relative orbits are strongly perturbed; for stars with relative velocity $v$ and mass $M$, the three stars need to pass within a distance of $\sim$ $G M / v^{2}$ of each other). In such close triple encounters, the interchange of energy within the system will in general cause one of the stars to acquire more kinetic energy at the expense of hardening the relative orbit of the other two. The repetitive action of such encounters causes the cluster to become unbound (the total energy, in this case, is of course conserved, and the kinetic energy of the escaping stars is released by the formation of a binary star system). Numerous simulations of the few body problem for a system of gravitating point masses (e.g. van Albada 1968; Harrington 1975; Valtonen \& Mikkola 1991; Sterzik \& Durisen 1995,1998 ) have shown that the resulting binary usually contains the two most massive cluster members. As pointed out by McDonald \& Clarke (1993), the implication of this result is that if stars form in this way, low mass stars and, particularly, brown dwarfs, should be less likely to be found in binary systems.

In reality, the situation explored by such Nbody simulations (whereby the stars have pre-assigned masses and interact through purely point mass gravitational interactions) is very artificial, and the system should instead be investi- 
gated as an initially gas dynamical one. Gas (whether distributed or in the form of circumstellar disks) modifies the dynamical decay process, since dissipation provides an additional channel for the redistribution of energy in the system (Clarke \& Pringle 1991, McDonald \& Clarke 1995). In addition, distributed gas (if it contains a significant fraction of the initial cluster mass) shapes the stellar masses as it is differentially accreted on to the stars as the cluster dissolves. Pilot simulations have shown that this situation is highly inequitable, and that even if the cluster contains protostars of initially equal masses, the interplay of gas dynamics and few body stellar dynamics produces a large dynamic range of final stellar masses (Bonnell et al. 1997, 2001).

Reipurth \& Clarke (2001) first explicitly linked the small N cluster scenario to the production of brown dwarfs. They emphasized that in the presence of gas, it is not merely the case that brown dwarfs are ejected because they are of low mass (as is the case in purely Nbody calculations) but that instead brown dwarfs are of low mass because they are prematurely ejected. In this scenario, the final mass of a star (or brown dwarf) is intimately linked to its dynamical and accretion history and one would therefore anticipate correlations between an object's mass and other characteristics (such as its binarity, circumstellar environment and kinematics). In particular, if brown dwarfs indeed form within cores whose total mass considerably exceeds that of a typical brown dwarf, then objects that end up as brown dwarfs must have suffered an ejection event (or, more probably, a sequence of close encounters culminating in one which imparted them with positive energy).

The typical length scale of such close encounters, $D$, may be imprinted on the resulting brown dwarfs in several ways. Firstly, the ejection speed is roughly related to the orbital velocity of the dwarf during close encounter; if the typical object in the cluster has the mass of a low mass star, then the ejection speed may be crudely parameterized as $15 D_{A U}^{-0.5}\left[\mathrm{~km} \mathrm{~s}^{-1}\right]$, where $D_{A U}$ is the close encounter distance in AU (Armitage \& Clarke 1997). Secondly, it is unlikely that the dwarf would be able to take with it a reservoir of circumstellar material with a size scale exceeding $D$. This limits both the mass and initial radius of disks in ejected objects. Subsequent viscous evolution can cause the disk to re-expand, but its lifetime is likely to be reduced as a result of the encounter. Thirdly, the object cannot be ejected as a binary if $D$ is less than or comparable to the binary separation. Thus one would not expect wide brown dwarf binaries (with separations greater than $\sim D$ ) to be produced by this mechanism.

Further quantification of these trends requires detailed numerical simulations. Recently, Bate (see contribution this volume) has placed such interactions within the context of a star forming molecular cloud. In these simulations (see Bate 2002, and Bate, Bonnell, \& Bromm 2002), supersonic turbulence produces a system of sheets and filaments of compressed gas, whose further fragmentation gives rise to a number of small $\mathrm{N}$ clusters. These clusters undergo the processes described above, although their evolution is more complex since each cluster is no longer a 'closed box' (as in previous studies) but may experience both further infall of gas as well as additional interactions as mini-clusters collide with each other (star formation activity is centered on the filaments in this simulation, so that cluster-cluster collisions tend to occur at the intersections of filaments as clusters fall along the filaments). The stars and brown dwarfs produced in the 
simulation (about 20 of each) all initially form at a mass scale of $\sim 10$ Jupiter masses, which is the minimum Jeans mass allowed by the equation of state employed. Their final masses, which roughly conform to the observed IMF in the stellar regime, are thus entirely set by the process of competitive accretion within a small $\mathrm{N}$ cluster environment.

A complementary investigation led by Delgado-Donate (see contribution this volume) has instead focused on multiple random realizations of small $\mathrm{N}$ clusters as isolated hydrodynamic systems. By considering isolated clusters, these simulations obviously cannot follow the cluster-cluster interactions that are a feature of the whole cloud simulations of Bate. In return, the computational economy effected by not modeling the larger cloud environment (about a factor of 6 in terms of CPU hours per star produced) allows one to obtain statistically significant numbers of stars for each initial condition and thus to explore the connection between initial conditions and resultant stellar properties. Furthermore, in contrast to Bate's simulations, these isolated simulations have been integrated as Nbody systems following the accretion of the gas, until they have decayed into stable hierarchical multiples and single stars. In this way, these simulations can shed some light on the properties of wide binaries, which remain as non-hierarchical multiples in Bate's simulations. Preliminary results from this investigation suggest that the statistical properties of the resulting stars and binaries are not unduly sensitive to the power spectrum of perturbations employed in the initial conditions; this suggests that the combination of fragmentation and competitive accretion may produce a rather robust set of stellar parameters, weakly dependent on the detailed environment.

\section{Observational Consequences of the Ejection Model}

In the following we summarize a number of observations, which may cast light on the validity of the ejection model.

Brown Dwarfs near Class 0 Sources.

The bulk of the mass of a newborn star is accumulated during its embedded phase, which lasts $\sim 10^{5} \mathrm{yr}$. Such nascent objects are seen as Class 0 and Class I sources. The strong outflow activity of Class 0 sources suggest that it is during this evolutionary stage that a star builds up most of its mass. It follows that if the accretion process for a stellar embryo should be interrupted early enough to limit its mass to substellar values, the dynamical interactions leading to break-up most likely occur during the Class 0 phase. How long time a newborn brown dwarf will be observable in the vicinity of an embedded source will then depend primarily on the ejection velocity. Assume that a newborn brown dwarf is observed at a time $t$ (years) after ejection, that it is moving with a space velocity $v(\mathrm{~km}$ per sec) at an angle $\alpha$ to the line of sight, and that it is located in a star forming region at a distance $d$ (parsecs). The projected separation $s$ in arcseconds from the embedded source will then be $s=0.21 v t d^{-1} \sin \alpha$. As an example, consider a newborn brown dwarf in a nearby cloud $(d \sim 130 p c)$ moving at an angle of $60^{\circ}$ to the line of sight with a space velocity of $1 \mathrm{~km} \mathrm{~s}^{-1}$. After $10^{5} \mathrm{yr}$, when the embedded phase of the original Class 0 object is about to end, the brown dwarf is already 140 arcsec away, with no obvious connection to its site of birth. Furthermore, half of all ejected brown dwarfs will move into the cloud in which 
they were born, appearing as very weak infrared sources, thus making their detection more difficult. Altogether, if sufficiently many Class 0 and perhaps Class I sources are surveyed, a number of newborn brown dwarfs could be found. A likely brown dwarf candidate was found 4 arcsec from the deeply embedded driving source of the HH 111 jet (Reipurth et al. 1999).

Kinematics of Brown Dwarfs.

If brown dwarfs are ejected stellar embryos, they should carry kinematic signatures reflecting their origin in small multiple systems. The velocity dispersion of young stars is generally assumed to reflect the turbulent velocity of the gas out of which they formed, that is, their velocities are expected to be of the order of a $\mathrm{km}$ per second. If all stars are formed in small multiple systems, as suggested by Larson (1972), then an additional velocity component comes from the velocities attained when the cluster dissolves and the members drift apart. The velocity that an ejected member acquires is comparable to the orbital speed attained at pericenter in the close triple encounter. Realistic numerical simulations including gas dynamics have been carried out by Delgado-Donate and Clarke (see their contribution in this volume), and they conclude that typical velocities of brown dwarfs are mostly less than $2 \mathrm{~km} \mathrm{~s}^{-1}$. One-dimensional velocities (radial or tangential) are correspondingly smaller. This is so close to the general turbulent velocity dispersion in molecular clouds that it will be difficult to test the ejection scenario kinematically. Further, Delgado-Donate and Clarke find that there is no dependence of the emergent speed on stellar mass amongst single ejected stars, although they find (in common with Sterzik \& Durisen 1998) that binary systems emerge with much lower velocities. Since the binary fraction is seemingly higher among stars than brown dwarfs, this would translate into an effective mass dependence of the emergent velocity.

Most brown dwarfs are, like stars, born within larger scale clusters. With the velocities quoted above, most stars and brown dwarfs would be retained within typical open clusters, but, after a crossing time or so, the distribution of brown dwarfs would be more extended due to their higher initial velocity dispersion. However, after about $10^{8}$ years, the velocities of cluster members are dominated by dynamical relaxation, which causes the brown dwarf distribution to be somewhat more distended than the stars (de la Fuente Marcos \& de la Fuente Marcos 2000, Adams et al. 2002), irrespective of the initial velocities with which they are injected into the cluster (Moraux et al., in prep.). A proper test of the ejection scenario is made only by finding a halo of brown dwarfs around clusters small enough and/or young enough that relaxation has not yet dominated their kinematics, but the size of the expected effect may be marginal. Finally, we note that kinematics of isolated old brown dwarfs no longer reflect conditions at the time of birth, since most will have evaporated from clusters.

Infrared Excesses of Brown Dwarfs.

The members of newborn multiple systems are likely to be surrounded by substantial amounts of circumstellar matter. However, as a prelude to dynamical ejection, a brown dwarf must go through a close triple encounter, which will prune its circumstellar disk. Free-floating brown dwarfs may therefore have smaller reservoirs from which they can accrete and form planets. Armitage \& Clarke (1997) showed that disk truncation in T Tauri stars could promote the rapid decline of classical $\mathrm{T}$ Tauri characteristics and, in analogy, we would 
therefore expect that ejected brown dwarfs will display the signatures of infall and outflow for a more limited time. But if encountered at an early enough age, a brown dwarf should show just the same indicators of disk accretion as any young star. In particular it should be stressed that the detection of nearinfrared excesses in brown dwarfs, and thus the presence of circumstellar disk material, is not at variance with the ejection hypothesis. Excesses at nearinfrared wavelengths refer to the inner edges of disks at distances of only a few stellar radii from the central object, regions which are unaffected by the disk truncation. If a brown dwarf disk is truncated at a radius of, say, $20 \mathrm{AU}$ (as is typical in the simulations of Bate 2002), then the observed spectral energy distribution is affected only at wavelengths longer than about $40 \mu \mathrm{m}$ (Natta \& Testi 2001; Natta et al. 2002). The near- and mid-infrared energy distributions are, however, sensitively dependent on the geometry of the disk, in particular the flaring angle, about which very little is known for brown dwarfs (see also Testi et al. in this volume).

The Brown Dwarf Desert.

The "brown dwarf desert" refers to the long known fact that brown dwarfs are only rarely found as close (less than $3 \mathrm{AU}$ ) companions to low mass stars (on these scales the incidence of brown dwarf companions to stars with masses of $0.5 \mathrm{M}_{\odot}$ or more does not exceed $1 \%$ ). Recently, however, Gizis et al. (2001) have demonstrated that at large separations (greater than $1000 \mathrm{AU}$ ) one commonly finds brown dwarf companions to normal stars.

The ejection hypothesis readily explains the absence of brown dwarfs close to normal stars, because of the combination of two effects. As is well known from pure N-body simulations of small $\mathrm{N}$ clusters, exchange interactions tend to substitute more massive cluster members into binaries that temporarily contain only low mass components. But, as shown in gas dynamical simulations, continued accretion tends to equalize binary mass ratios (Artymowicz 1983, Bate 2000), so that a brown dwarf companion may not remain as such unless the binary is swiftly removed from the reservoir of gas that feeds it. Only under special circumstances (see Reipurth \& Clarke 2001) will dynamical interactions directly produce a brown dwarf as a close companion to a star (but it may arise through subsequent orbital evolution if the two components have circumstellar material that interacts). On the other hand, the ejection hypothesis readily explains the presence of brown dwarfs as distant companions to stars, simply because not all ejections lead to an unbound system.

Of course, it is possible that the brown dwarf desert may not be a remnant of the formation process, but could result from orbital migration (Armitage \& Bonnell 2002).

\section{Brown Dwarf Binaries.}

An increasing number of brown dwarfs have been found to be binaries (e.g. Martín, Brandner, \& Basri 1999). The distribution of separations in brown dwarf binaries contains important memories of their formation, which any theory of brown dwarf formation must be able to reproduce. It is notable that, up to now, only rather close brown dwarf binaries have been discovered. Pairs with separations of many hundreds of AU are not seen, although the observing techniques have no obvious bias against their detection. This is equally true for 
brown dwarfs in clusters and in the field, perhaps suggesting that we are not seeing an effect of their environment, but a result of their formation process.

From the perspective of the ejection hypothesis, there are two aspects of this problem: the formation of binary embryos, and the survival of the nascent binary during its ejection. The latter clearly militates against wide binaries, since they would be torn apart in a close triple encounter, thus being consistent with the tentative absence of observations of wide brown dwarf pairs.

The formation of binary embryos is more problematical. McDonald \& Clarke (1993) and Sterzik \& Durisen (1998) showed that for purely point-mass dynamical interactions, binaries made up of two brown dwarfs would essentially never be formed. However, stellar embryos in small multiple systems are likely to be associated with substantial circumstellar material, offering a source of dissipation that can create such low mass pairs. A further problem, however, is that such pairs do not generally remain as brown dwarfs but instead accrete over the hydrogen burning mass limit. As a result, brown dwarf binaries are rather rare in the simulations of Bate (2002) and Delgado-Donate \& Clarke (see this volume).

\section{Binarity of Stellar Primaries.}

Evidently, for a stellar embryo to be ejected as a brown dwarf requires the presence of at least three bodies that interact. When the third member is ejected, either into a distant but bound orbit or as an unbound object, the two remaining components are bound more closely in a harder binary. It is of great interest to examine the primaries in wide binary systems with a brown dwarf companion, since the ejection hypothesis posits that such primaries should themselves be close binaries. Depending on the distance to the object, such binaries may be resolved with adaptive optics techniques, or with spectroscopic techniques.

For close binaries with a low mass primary and a brown dwarf companion there is the possibility that the entire binary was ejected, just as brown dwarf pairs can be ejected, but current simulations suggest that this is not commonly happening. In such cases the primary is obviously not expected to be itself a binary. Clearly the study of the binarity of primaries is likely to be an important test of the ejection hypothesis. For a more detailed discussion, see Sterzik \& Durisen in this volume.

The Rotation Rates of Brown Dwarfs.

If disks around brown dwarfs are truncated by the ejection process, then this may affect the rotation rate of young brown dwarfs, since magnetic coupling to an accretion disk is the most popular mechanism for explaining the relatively modest rotation rates of young stars (e.g. Königl 1991, Cameron \& Campbell 1993, Armitage \& Clarke 1996). It is not, however, clear whether the interactions that we are positing here (within $\sim 20 \mathrm{AU}$ ) are close enough to release the protodwarf from its disk brake. Detailed simulations of magnetic braking in stars suggest that the disk brake only becomes ineffective at very low disk masses (Armitage \& Clarke 1996). The value of this critical disk mass depends on the moment of inertia of the star and is higher at early times when the star is more extended. Thus, as discussed by Clarke \& Bouvier (2000), if disk stripping is to have any effect on stellar rotation, it must occur at early times, which is certainly an expectation in the dynamical decay scenario considered here. In conclusion, the sign of the possible effect is clear - i.e. that objects whose disks are pruned 
would tend to rotate faster. What is less clear is whether the interactions we expect in mini-clusters would be close enough to make a substantial difference to the rotation rates of young brown dwarfs.

\section{What do Newborn Brown Dwarfs look like?}

It follows from the preceding discussion that if brown dwarfs are ejected stellar embryos we should, at least in principle, be able to find brown dwarfs near Class 0 sources which are extremely young, even less than $10^{5} \mathrm{yr}$ old. From an observational point of view it may, however, not be so clear what we should be looking for to identify such newborn brown dwarfs. Models for relatively old brown dwarfs are in excellent agreement with observations. But, as stressed by Baraffe et al. (2002), for young objects the models become very sensitive to the initial conditions, e.g. the initial radius. And for ages less than about $1 \mathrm{Myr}$ the input conditions are still so important for the models that they cannot be meaningfully compared to observations (see also Baraffe et al. in this volume).

So what do we look for when we try to find the youngest brown dwarfs? The boundary between stellar and substellar objects at the age of nearby young associations is at a spectral type around M6, so we may expect to see strong molecular bands. However, if a stellar embryo has very recently been exposed to heavy infall, its outermost layers may be disturbed and heated at least for about $10^{4}$ years after ejection, which is the Kelvin-Helmholtz timescale for young brown dwarfs. During this period the surface temperatures are possibly high enough to dissociate molecules. Also, even though the outer parts of the circumstellar disk of an ejected brown dwarf will be truncated, the inner parts are likely to be extremely perturbed by the close triple encounter (e.g. Clarke \& Pringle 1991), most likely leading to active accretion. So although we expect newborn brown dwarfs to have very low luminosities, the observed luminosities may be increased through contributions from accretion. Spectroscopically, we may expect that the very youngest brown dwarfs are heavily veiled objects, perhaps obscuring all absorption features, and showing strong $\mathrm{H} \alpha$ emission. And if, as seems likely, the stellar embryos also have magnetic fields, we may expect the very youngest and heavily accreting brown dwarfs to have small jets, which may be visible in forbidden lines and as thermal radio jets. These are, however, signatures of youth commonly found among very young low mass stars, so we may find that identifying newborn brown dwarfs as bona fide substellar objects could be a difficult task.

Acknowledgments. We are grateful to Isabel Baraffe, Matthew Bate, Alan Boss, Eduardo Delgado-Donate, and Chris Tout for useful discussions.

\section{References}

Adams, T., Davies, M.B., Jameson, R.F., Scally, A. 2002, MNRAS 333, 547

Armitage, P.J., Bonnell, I.A. 2002, MNRAS, 330, 11

Armitage, P.J., Clarke, C.J. 1996, MNRAS 280, 458

Armitage, P.J., Clarke, C.J. 1997, MNRAS, 285, 540

Artymowicz, P. 1983, Acta Astronomica 33, 223 
Artymowicz, P., Lubow, S.H. 1996, ApJ, 467, L77

Baraffe, I., Chabrier, G., Allard, F., Hauschildt, P. 2002, A\&A, 382, 563

Bate, M.R. 2000, MNRAS, 314, 33

Bate, M.R., Clarke, C.J., McCaughrean, M.J. 1998, MNRAS, 297, 1163

Bate, M.R. 2002, MNRAS, in press

Bate, M.R., Bonnell, I.A., Bromm, V. 2002, MNRAS, 332, L65

Bonnell, I.A., Bate, M.R., Clarke, C.J. \& Pringle, J.E. 1997, MNRAS 323, 785

Bonnell, I.A., Bate, M.R., Clarke, C.J., Pringle, J.E. 2001, MNRAS, 323, 785

Boss, A.P. 2001, ApJ, 551, L167

Cameron, A.C., Campbell, C.G. 1993, A\&A 274, 309

Clarke, C.J., Pringle, J. 1991, MNRAS, 249, 588

Clarke, C. J., Bouvier, J. 2000, MNRAS 319, 457

de la Fuente Marcos, R., de la Fuente Marcos, C. 2000, ApSS 271, 127

Elmegreen, B.C. 1999, ApJ, 522, 915

Gizis, J.E., Kirkpatrick, J.D., Burgasser, A., Reid, I.N., Monet, D.G., Liebert, J., Wilson, J.C. 2001, ApJ, 551, L163

Harrington, R.S. 1975, AJ, 80, 1081

Königl, A. 1991, ApJ 370, 39

Larson, R.B. 1972, MNRAS, 156, 437

Li, Z.-Y. 2002, ApJ, 574, L159

Lin, D., Laughlin, G., Bodenheimer, P., Rozyczka, M. 1998, Science, 281, 2025

Martín, E.L., Brandner, W., Basri, G. 1999, Science, 283, 1718

McDonald, J.M., \& Clarke, C.J. 1993, MNRAS, 262, 800

McDonald, J.M., \& Clarke, C.J. 1995, MNRAS, 275, 671

Muench, A.A, Alves, J.A., Lada, C.J., Lada, E.A. 2001, ApJ, 558, L51

Natta, A., Testi, L. 2001, A\&A, 367, L22

Natta, A., Testi, L., Comerón, F., Oliva, E., D'Antona, F., Baffa, C., Comoretto, G., Gennari, S. 2002, A\&A, in press

Padoan, P., Nordlund, A. 2002, in press

Scally, A., Clarke, C.J. 2002, MNRAS, 334, 156

Reipurth, B. 2000, AJ, 120, 3177

Reipurth, B., Clarke, C.J. 2001, AJ, 122, 432

Reipurth, B., Yu, K.C., Rodríguez, L.F., Heathcote, S., Bally, J. 1999, A\&A, 352, L86

Sterzik, M.F., Durisen, R.H. 1995, A\&A, 304, L9

Sterzik, M.F., Durisen, R.H. 1998, A\&A, 339, 95

Valtonen, M., Mikkola, S. 1991, ARA\&A, 29, 9

van Albada, T.S. 1968, Bull. Astron. Inst. Netherlands, 19, 479

Whitworth, A., Zinnecker, H. 2002, in press 